\title{
Forbidden paths and cycles in ordered graphs and matrices
}

\author{
János Pach Gábor Tardos
}

\begin{abstract}
At most how many edges can an ordered graph of $n$ vertices have if it does not contain a fixed forbidden ordered subgraph $H$ ? It is not hard to give an asymptotically tight answer to this question, unless $H$ is a bipartite graph in which every vertex belonging to the first part precedes all vertices belonging to the second. In this case, the question can be reformulated as an extremal problem for zero-one matrices avoiding a certain pattern (submatrix) $P$. We disprove a general conjecture of Füredi and Hajnal related to the latter problem, and replace it by some weaker alternatives. We verify our conjectures in a few special cases when $P$ is the adjacency matrix of an acyclic graph and discuss the same question when the forbidden patterns are adjacency matrices of cycles. Our results lead to a new proof of the fact that the number of times that the unit distance can occur among $n$ points in the plane is $O\left(n^{4 / 3}\right)$.
\end{abstract}

\section{Introduction}

A simple graph $G$ with a linear ordering on its vertex set $V(G)$ is called an ordered graph. The edge set of $G$ is denoted by $E(G)$. In the spirit of the fundamental problem of Turán-type extremal graph theory [3], one can raise the following general question. What is the maximum number $\mathrm{ex}_{<}(n, H)$ of edges that an ordered graph on $n$ vertices can have without containing a (not necessarily induced) subgraph isomorphic to a fixed ordered graph $H$ ? The ordering of the vertices is inherited by the subgraphs. An isomorphism between two ordered graphs is an isomorphism between the underlying unordered graphs that respects the ordering of the vertices. If a graph does not contain $H$ as an ordered subgraph, it is called $H$-free. We assume $H$ has at least one edge.

Define the interval chromatic number $\chi_{<}(H)$ of an ordered graph $H$, as the minimum number of intervals the (linearly ordered) vertex set of $H$ can be partitioned into, so that no two vertices belonging to the same interval are adjacent in $H$. By a simple application of the Erdös-Stone theorem [7], one can easily describe the asymptotic behavior of $\operatorname{ex}_{<}(n, H)$, unless $\chi_{<}(H)=2$. See also [5] for a similar result and proof.

Theorem 1 For any ordered graph $H$, the maximum number of edges that an $H$-free ordered graph with $n$ vertices can have satisfies

$$
\operatorname{ex}_{<}(n, H)=\left(1-\frac{1}{\chi_{<}(H)-1}\right)\left(\begin{array}{l}
n \\
2
\end{array}\right)+o\left(n^{2}\right) .
$$

Proof. Let $G$ be an $H$-free ordered graph with $n$ vertices. Let $m=|V(H)|, \chi=\chi_{<}(H)$, and let $K_{\chi}(m)$ denote the unordered $\chi$-partite complete graph with $m$ vertices in each of its vertex classes $V_{1}, \ldots, V_{\chi}$. It follows from the Erdös-Stone theorem that if the unordered graph obtained from $G$ by disregarding the ordering contains no $K_{\chi}(m)$, then its number of edges is at most $\left(1-\frac{1}{\chi-1}\right)\left(\begin{array}{l}n \\ 2\end{array}\right)+$ $o\left(n^{2}\right)$. 
Therefore, it is sufficient to show that in any ordering of $K_{\chi}(m)$ there is an ordered subgraph isomorphic to $H$. To see this, let $v_{i}^{1}, \ldots, v_{i}^{m}$ be the elements of $V_{i}$ in increasing order $(1 \leq i \leq \chi)$. Partition the vertex set of $H$ into $\chi$ independent intervals. For $0 \leq j \leq \chi$, let $m_{j}$ stand for the total size of the first $j$ intervals. Define a permutation $\pi$ on $\{1, \ldots, \chi\}$ as follows. Let $\pi(1)$ be the index $i$ for which $v_{i}^{m_{1}}$ is the smallest. Assume that we have already defined $\pi(1), \ldots, \pi(j-1)$. Let $\pi(j)$ be the index $i$ different from the previous ones for which $v_{i}^{m_{j}}$ is the smallest. Now we can easily give an order preserving embedding of $H$ into $K_{\chi}(m)$ : if the $j$ th smallest vertex of $H$ belongs to the $i$ th interval (that is, we have $m_{i-1}<j \leq m_{i}$ ), then map it to $v_{\pi(i)}^{j}$.

This theorem naturally extends to families $\mathcal{H}$ of forbidden ordered subgraphs with $\chi_{<}(\mathcal{H}):=$ $\min \left\{\chi_{<}(H) \mid H \in \mathcal{H}\right\}$.

Note that the interval chromatic number is easily computable. Indeed, by a simple greedy algorithm one can efficiently find an optimal partition of the vertex set of $H$ into $\chi_{<}(H)$ independent intervals. This is in sharp contrast with the fact that even the approximation of the usual chromatic number of a graph is an NP-hard task.

As shown by Theorem 1, determining the maximum number of edges of an $H$-free ordered graph becomes more interesting when $\chi_{<}(H)=2$. In this special case, it is more convenient to restrict our attention to $H$-free ordered graphs $G$ which themselves have interval chromatic number 2 . The vertices of such a graph can be enumerated as $v_{1}<v_{2}<\ldots<v_{n}<v_{n+1}<\ldots<v_{n+m}$ so that every edge of $G$ connects some $v_{i}, i \leq n$ to a $v_{j}, j>n$. Let $A=A(G)$ be an $n \times m$ adjacency matrix whose rows and columns correspond to the vertices $v_{i}, i \leq n$ and $v_{j}, j>n$, respectively, and whose entry $a_{i, j-n}=1$ if $v_{i} v_{j}$ is an edge of $G$, and 0 otherwise. $A(G)$ is uniquely determined if $G$ has a unique decomposition into two independent intervals. This is the case, for example, if $G$ has no isolated vertices. Conversely, any $n \times m$ zero-one matrix $A$ gives rise to an ordered graph $G(A)$ with $\chi_{<}(G(A)) \leq 2$, whose vertices correspond to the rows and columns of $A$, and the adjacencies between the two kinds of vertices depend on the corresponding entry of $A$. We always have $G(A(G))=G$.

The weight $w(A)$ of a zero-one matrix $A$ is the number of its 1 entries. A zero-one matrix of positive weight is called a pattern. Following [9], we say that a zero-one matrix $A$ contains a pattern $P$ if $P$ is a submatrix of $A$ or if $P$ can be obtained from a submatrix of $A$ by changing some 1 entries to 0 . The corresponding submatrix of $A$ is said to represent $P$. Notice that we can delete some rows or columns of $A$ to find the submatrix $P$, but we are not allowed to permute the remaining rows and columns. If $A$ does not contain $P$, we say that $A$ avoids $P$. Let ex $(n, m, P)$ denote the maximum weight of an $n \times m$ zero-one matrix that avoids $P$. For simplicity, write $\operatorname{ex}(n, P)$ instead of ex $(n, n, P)$. If a family $\mathcal{P}$ of patterns is forbidden, we use $\operatorname{ex}(n, \mathcal{P})$ to denote the corresponding maximum weight. The problem of estimating these functions for various patterns has been considered in $[1,2,8,9,11,15]$.

Let $G$ and $H$ be two ordered graphs with interval chromatic number 2, and assume that $H$ has a unique decomposition into two independent intervals. Then $G$ is $H$-free if and only if $A(G)$ avoids $A(H)$. Therefore, if $G$ is $H$-free and the first and second intervals in its decomposition consist of $n$ and $m$ elements, respectively, then its number of edges satisfies

$$
|E(G)| \leq \operatorname{ex}(n, m, A(H)) .
$$

If we only assume that $\chi_{<}(H)=2$, but there is no assumption on the host graph $G$, then the situation is somewhat more complicated. Nevertheless, in Section 2 we prove the following general result linking the solutions of the extremal problems for graphs and for patterns (matrices). 
Theorem 2 Let $H$ be an ordered graph with interval chromatic number 2, which has a unique decomposition into two intervals that are independent sets. Then we have

$$
\operatorname{ex}(\lfloor n / 2\rfloor, A(H)) \leq \operatorname{ex}_{<}(n, H)=O(\operatorname{ex}(n, A(H)) \log n) .
$$

Moreover, if $\operatorname{ex}(n, A(H))=O\left(n^{c}\right)$ holds for some $c>1$, then we have $\mathrm{ex}_{<}(n, H)=O\left(n^{c}\right)$.

We conjecture that if $H$ is an ordered tree of interval chromatic number 2 , then $\operatorname{ex}_{<}(n, H)$ is only at most slightly superlinear (Conjecture 1). In Section 3, we verify this statement in several special cases.

In Section 4, we consider the case when $H$ is an ordered cycle (of even length) with $\chi_{<}(H)=2$. It is well known (see [3]) that there are (unordered) graphs with $n$ vertices and with at least constant times $n^{1+\frac{1}{2 k}}$ edges that contain no cycle of length $2 k$ or shorter. Therefore, by Theorem 2 , in this case the order of magnitude of $\operatorname{ex}_{<}(n, H)$ is the same as that of the solution of the corresponding matrix problem. In Section 4, we analyze the latter version of the question.

We call a sequence $C=\left(p_{0}, p_{1}, \ldots, p_{2 k}\right)$ of positions in a matrix $A$ an orthogonal cycle if $p_{0}=p_{2 k}$ and the positions $p_{2 i}$ and $p_{2 i+1}$ belong to the same row, while the positions $p_{2 i+1}$ and $p_{2 i+2}$ belong to the same column, for every $0 \leq i<k$. If the entry of $A$ in positions $p_{i}$ is 1 for all $0 \leq i \leq 2 k$, then $C$ is said to be an orthogonal cycle of $A$. Notice that, for any zero-one matrix $A$, each cycle of $G(A)$ (with a starting point and an orientation) corresponds to an orthogonal cycle of $A$. In general, an orthogonal cycle of $A$ corresponds to a walk in $G(A)$ that starts and ends at the same vertex.

Given a position $p=(i, j)$ of the matrix $A$ and an orthogonal cycle $C=\left(p_{0}, p_{1}, \ldots, p_{2 k}\right)$, define $C(i, j)$ to be the number of times that the possibly self-intersecting polygon $p_{0} p_{1} \ldots p_{2 k}$ encircles (in the counter-clockwise direction) a point $p^{\prime}=(i+1 / 2, j+1 / 2)$ of the plane. Here we interpret the position $(i, j)$ in a matrix as the point $(i, j)$ or the Euclidean plane. Notice that this convention is against the tradition of writing the first row of a matrix on top. Formally, let $P(i, j)$ be the set of positions $\left(i^{\prime}, j^{\prime}\right)$ with $i^{\prime}>i$ and $j^{\prime}>j$, and set

$$
C(i, j)=\left|\left\{0<l \leq k: p_{2 l} \in P(i, j)\right\}\right|-\left|\left\{0<l \leq k: p_{2 l-1} \in P(i, j)\right\}\right| .
$$

An orthogonal cycle is said to be positive if $C(i, j) \geq 0$ for every pair $(i, j)$ and $C(i, j)$ is strictly positive for at least one such pair. A collection $\mathcal{C}$ of orthogonal cycles is called positive if $\sum_{C \in \mathcal{C}} C(i, j) \geq 0$ for every $(i, j)$ and there exists at least one $(i, j)$ for which this sum is positive.

Let $G$ be an ordered graph with interval chromatic number 2. It is easy to check that, for any cycle of length 4 , the corresponding entries of the adjacency matrix $A(G)$, with a proper orientation, form a positive orthogonal cycle. However, the entries of $A(G)$ assigned to the edges of a cycle of length 6 may or may not induce a positive orthogonal cycle. For obvious reasons, cycles of the former type are called noncrossing hexagons. Katz [10] proved that the maximum weight of an $n$ by $n$ zero-one matrix that avoids cycles of length four and noncrossing hexagons (or, equivalently, positive orthogonal cycles of length at most 6$)$ is $O\left(n^{\frac{3}{2}-\varepsilon}\right)$ for some $\varepsilon>0$. This is somewhat stronger than the trivial bound $O\left(n^{\frac{3}{2}}\right)$, which is tight when only 4-cycles are forbidden. Katz applied his result to measure-theoretic problems.

The main result of Section 4 is the following.

Theorem 3 The maximum weight of an $n$ by $n$ zero-one matrix containing no positive orthogonal cycle is $O\left(n^{4 / 3}\right)$. The order of magnitude of this bound cannot be improved. 
In fact, we prove a stronger result (Theorem 5) that provides several counterexamples to a conjecture of Füredi and Hajnal [9]. It also offers a new proof of the following well-known theorem of Spencer, Szemerédi, and Trotter [12].

Corollary 1 [12] The number of unit distance pairs determined by $n$ points in the plane is $O\left(n^{4 / 3}\right)$.

The proof of these facts, a counterexample to a related conjecture of Brass, Károlyi, and Valtr [5], as well as some concluding remarks are presented in Section 5.

\section{Ordered graphs vs. zero-one matrices}

First, we establish Theorem 2 connecting the extremal problems for ordered graphs and matrices. Roughly speaking, it shows that if we want to estimate the maximum number of edges that an $H$-free ordered graph of $n$ vertices can have, we do not lose much by restricting the search to ordered graphs with interval chromatic number 2. For the proof, we need two simple observations summarized in the following lemma. Throughout this paper, log always stands for logarithm of base 2 .

Lemma 1 (i) For any ordered graph $G$ of $n$ vertices, one can find edge disjoint subgraphs $G_{i}$ for $0 \leq i \leq\lceil\log n\rceil$ such that $E(G)=\cup_{i=0}^{\lceil\log n\rceil} E\left(G_{i}\right)$ and each connected component of $G_{i}$ has at most $\left\lceil n / 2^{i}\right\rceil$ vertices and interval chromatic number at most 2.

(ii) (Super-additivity) For any pattern $P$ and for any positive integers $n$ and $m$, we have

$$
\operatorname{ex}(n+m, P) \geq \operatorname{ex}(n, P)+\operatorname{ex}(m, P) .
$$

Proof. To show (i), denote the vertices of $G$ by $v_{0}, \ldots, v_{n-1}$. Let $G_{i}$ consist of all edges $v_{j} v_{k} \in E(G)$, for which $\left\lfloor 2^{i} j / n\right\rfloor=\left\lfloor 2^{i} k / n\right\rfloor$ but $\left\lfloor 2^{i+1} j / n\right\rfloor \neq\left\lfloor 2^{i+1} k / n\right\rfloor$. These subgraphs obviously meet the requirements.

To verify part (ii), we establish the super-additivity of the asymmetric version of the ex function:

$$
\operatorname{ex}\left(n_{1}+m_{1}, n_{2}+m_{2}, P\right) \geq \operatorname{ex}\left(n_{1}, n_{2}, P\right)+\operatorname{ex}\left(m_{1}, m_{2}, P\right) .
$$

Assume first that $P=\left(p_{i j}\right)$ has at least a single 1 entry in its first row, at least one 1 in its last row, and that the same holds for its first and last columns. Mark a 1 entry in the first row of $P$ red, a 1 entry in the last row of $P$ blue, and assume without loss of generality that the blue entry does not lie to the right of the red one. Let $A$ and $B$ be $n_{1} \times n_{2}$ and $m_{1} \times m_{2}$ zero-one matrices, respectively, that avoid $P$. Let us obtain the $\left(n_{1}+m_{1}\right) \times\left(n_{2}+m_{2}\right)$ matrix $C$ by putting $A$ and $B$ together as blocks along the main diagonal, and filling all the remaining positions by 0 . We claim that $C$ avoids $P$. Suppose not. If the red entry of $P$ is represented in block $B$ or the blue entry is represented in block $A$, then $B$ or $A$ would not avoid $P$, respectively. Thus, we can assume that the blue entry is represented in $B$, and the red entry is represented in $A$. However, in this case the blue entry lies to the right of the red one, which is impossible.

Suppose next that, e.g., the first row of $P$ contains no entry 1 . For any matrix $A$, let $A^{\prime}$ denote the matrix obtained from $A$ by removing its first row. Then $A$ contains $P$ if and only if $A^{\prime}$ contains $P^{\prime}$. Using this simple observation, it is not hard to see that $\operatorname{ex}(n, m, P)=\operatorname{ex}\left(n-1, m, P^{\prime}\right)+m$. This implies that super-additivity is inherited from $P^{\prime}$ to $P$. Therefore, it must hold for every pattern $P$. 
Proof of Theorem 2. The inequality $\operatorname{ex}_{<}(n, H) \geq \operatorname{ex}(\lfloor n / 2\rfloor, A(H))$ directly follows from the definitions: if $A$ is an $\lfloor n / 2\rfloor \times\lfloor n / 2\rfloor$ zero-one matrix not containing $A(H)$, then $G(A)$ is an $H$-free ordered graph on $2\lfloor n / 2\rfloor$ vertices whose number of edges coincides with the weight of $A$.

To prove the upper bound on $\operatorname{ex}_{<}(n, H)$, consider an ordered graph $G$ on $n$ vertices that does not contain $H$. Apply Lemma 1 (i) to partition the edges of $G$ into subgraphs $G_{i}$ satisfying the conditions. Since any nontrivial connected component $C$ of $G_{i}$ is $H$-free, the matrix $A(C)$ cannot contain $A(H)$. Thus, we have $e_{C} \leq \operatorname{ex}\left(n_{C}, A(H)\right)$, where $n_{C}$ and $e_{C}$ denote the number of vertices and the number of edges in $C$. If $\operatorname{ex}(n, A(H))=O\left(n^{c}\right)$ for some $c>1$, then summing these estimates over all $i$ and over all connected components $C$ of $G_{i}$, we obtain that $|E(G)|=O\left(n^{c}\right)$, as required. In the general case, summing over all connected components $C$ of a fixed $G_{i}$ and using the superadditivity property in Lemma 1 (ii) we can conclude that $\left|E\left(G_{i}\right)\right| \leq \operatorname{ex}(n, A(H))$, and hence $G$ has at most $(\log n+2) \operatorname{ex}(n, A(H))$ edges.

As shown by Theorem 2, there is little difference between the extremal problems for ordered graphs and for the corresponding zero-one matrices. In many cases, one cannot get rid of the logarithmic factor in the second inequality. Consider, for instance, the ordered graph $G_{4}$ with vertices $v_{1}<v_{2}<v_{3}<v_{4}$ and edges $v_{3} v_{1}, v_{1} v_{4}$, and $v_{4} v_{2}$. As an unordered graph, $G_{4}$ is a path of length 3. Now $A\left(G_{4}\right)$ is a $2 \times 2$ matrix consisting of three 1 entries and a 0 entry. It is easy to verify that $\operatorname{ex}\left(n, A\left(G_{4}\right)\right)=2 n-1$.

On the other hand, let $G$ be an ordered graph with vertices $v_{1}, \ldots, v_{n}$, where $v_{i}$ is connected to $v_{j}$ if and only if $|i-j|$ is a power of 2 . Clearly, $G$ is $G_{4}$-free and its number of edges is at least $n \log n-n$. Thus, in this case we have

$$
\operatorname{ex}_{<}\left(n, G_{4}\right) \geq n \log n-n \geq \frac{\log n}{4} \operatorname{ex}\left(n, A\left(G_{4}\right)\right) .
$$

We remark that Lemma 1 (ii) concerning the super-additivity of the function $\operatorname{ex}(n, P)$, does not extend to arbitrary families of forbidden patterns. For instance, let $\mathcal{P}$ be the family consisting of all $n \times n$ zero-one patterns of weight 1 . Clearly, we have $\operatorname{ex}(i, \mathcal{P})=i^{2}$ for $i<n$, but $\operatorname{ex}(i, \mathcal{P})=0$ for $i \geq n$.

\section{Trees}

We say that an ordered graph is acyclic if its underlying unordered graph contains no cycles. The aim of this section is to establish some partial results concerning the following conjecture.

Conjecture 1 For any acyclic ordered forbidden graph $H$ with interval chromatic number 2, we have $\operatorname{ex}_{<}(n, H) \leq n(\log n)^{O(1)}$.

Notice that, if true, this statement strongly characterizes acyclic ordered graphs $H$ with $\chi_{<}(H) \leq$ 2: for any other graph $H$, there exists $\varepsilon>0$ such that $\operatorname{ex}_{<}(n, H) \geq n^{1+\varepsilon}$. Indeed, in view of Theorem 1 , if $\chi_{<}(H) \geq 3$, the extremal function $\mathrm{ex}_{<}(n, H)$ is quadratic. On the other hand, if $H$ has a cycle of length $k$ (with any ordering), then its extremal function is at least as large as the maximum number of edges that a $C_{k}$-free unordered graph of $n$ vertices can have, which is $\Omega\left(n^{1+\frac{1}{k}}\right)$.

Conjecture 1 is stated with the upper bound $n(\log n)^{O(1)}$. We do not know, however, any counterexample to this conjecture with the stronger bound $O(n \log n)$, which has been proposed by Füredi and Hajnal [9]. It would also be interesting to prove a weaker form of the same statement, according to which $\operatorname{ex}_{<}(n, H)=O\left(n^{1+\varepsilon}\right)$ holds for any $\varepsilon>0$. 
In order to establish Conjecture 1 in some special cases, we need a couple of statements related to the corresponding problems for zero-one matrices.

Lemma 2 Assume that the last column of a pattern $P$ contains a single 1 entry, and let $P^{\prime}$ denote the pattern obtained from $P$ by removing this column. Then we have

$$
\operatorname{ex}(n, P)=O\left(\sum_{i \geq 0} 2^{i} \operatorname{ex}\left(\left\lfloor n / 2^{i}\right\rfloor, P^{\prime}\right)\right),
$$

and, consequently, $\operatorname{ex}(n, P)=O\left(\operatorname{ex}\left(n, P^{\prime}\right) \log n\right)$. Furthermore, if $\operatorname{ex}\left(n, P^{\prime}\right)=O\left(n^{c}\right)$ holds for some $c>1$, then we have $\operatorname{ex}(n, P)=O\left(n^{c}\right)$.

Proof. It is sufficient to prove the first part of the statement, because it implies the last two claims, just like in the proof of Theorem 2.

Let $A$ be an $n \times m$ zero-one matrix, which avoids $P$ and whose weight is maximum, that is, we have $w(A)=\operatorname{ex}(n, m, P)$. Assume that $m$ is even and consider the submatrix $A_{1}$ of $A$ formed by all rows of $A$ that have no 1 entry in their last $m / 2$ positions. Let $A_{2}$ be the submatrix of $A$ formed by the remaining rows $A$. Denote by $n_{1}$ and $n_{2}$ the number of rows in $A_{1}$ and $A_{2}$, respectively, so that we have $n_{1}+n_{2}=n$. Furthermore, for $i=1$ and 2 , let $A_{i 1}$ and $A_{i 2}$ denote the submatrices of $A_{i}$ formed by the first $m / 2$ and by the last $m / 2$ columns of $A_{i}$, respectively. Clearly, we have

$$
\operatorname{ex}(n, m, P)=w(A)=w\left(A_{11}\right)+w\left(A_{12}\right)+w\left(A_{21}\right)+w\left(A_{22}\right),
$$

where $w\left(A_{12}\right)=0$ holds, by definition. Since the other three matrices on the right-hand side are submatrices of $A$, they all avoid $P$. Therefore, $w\left(A_{11}\right) \leq \operatorname{ex}\left(n_{1}, m / 2, P\right)$ and $w\left(A_{22}\right) \leq \operatorname{ex}\left(n_{2}, m / 2, P\right)$. As for $A_{21}$, it also avoids the pattern $P^{\prime}$. Indeed, if $A_{21}$ had a submatrix representing $P^{\prime}$, adding to it a column of $A_{22}$ we would obtain a representation of $P$. Thus, we have $w\left(A_{21}\right) \leq \operatorname{ex}\left(n_{2}, m / 2, P^{\prime}\right)$. This yields

$$
\operatorname{ex}(n, m, P) \leq \operatorname{ex}\left(n_{1}, m / 2, P\right)+\operatorname{ex}\left(n_{2}, m / 2, P\right)+\operatorname{ex}\left(n_{2}, m / 2, P^{\prime}\right) .
$$

Assume now that $m=2^{k}$. Applying the above bound recursively $k$ times, we conclude that

$$
\operatorname{ex}(n, m, P) \leq \sum_{i=1}^{k} \sum_{j=1}^{2^{i-1}} \operatorname{ex}\left(n_{i j}, m / 2^{i}, P^{\prime}\right)+n,
$$

where the nonnegative integers $n_{i j}$ satisfy that $\sum_{j=1}^{2^{i-1}} n_{i j} \leq n$, for any $1 \leq i \leq k$.

Every $n \times m$ matrix avoiding $P^{\prime}$ can be partitioned into $\lceil n / m\rceil$ submatrices of size at most $m \times m$, so that we have $\operatorname{ex}\left(n, m, P^{\prime}\right) \leq\lceil n / m\rceil \operatorname{ex}\left(m, P^{\prime}\right)$. This, in turn, implies

$$
\operatorname{ex}(n, P) \leq \sum_{i=1}^{k}\left(2^{i}+2^{i-1}\right) \operatorname{ex}\left(n / 2^{i}, P^{\prime}\right)+n,
$$

if $n=2^{k}$. Thus, the first statement of the lemma holds for powers of 2, and, by the monotonicity of the ex function, it is also true for all other values of $n$.

There are several examples showing that the logarithmic factor in Lemma 2 cannot be always removed. Let $F=\left(\begin{array}{lll}1 & 1 & 0 \\ 1 & 0 & 1\end{array}\right)$. Füredi [8] and Bienstock-Győri [2] proved that $\operatorname{ex}(n, F)=\Theta(n \log n)$, 
while Tardos [15] found the sharper estimate $\operatorname{ex}(n, F)=n \log n+O(n)$. On the other hand, as mentioned before, the pattern $F^{\prime}$ obtained from $F$ by removing its last column satisfies the equation $\operatorname{ex}\left(n, F^{\prime}\right)=2 n-1$.

Applying Theorem 2 once and Lemma 2 several times, one can verify Conjecture 1 for a large class of graphs. By symmetry, one can apply Lemma 2 to eliminate the first column or the first (last) row of a pattern, provided that it has a single 1 entry. In particular, the conjecture holds for all perfect matchings, i.e., ordered graphs $H$ whose adjacency matrix $A(H)$ has precisely one 1 in each of its rows and columns. In fact, in this case, improving some earlier results of Alon and Friedgut [1], Marcus and Tardos [11] established a linear upper bound on $\operatorname{ex}(n, A(H))$. The smallest ordered graphs $H$ for which Conjecture 1 cannot be proved in this way are paths of length 5 whose adjacency matrix $A(H)$ is

$$
\left(\begin{array}{lll}
1 & 0 & 1 \\
1 & 0 & 0 \\
0 & 1 & 1
\end{array}\right),
$$

or one of the three other matrices that can be obtained from this one by rotation (or reflection).

Before proving Conjecture 1 for this path and for many other patterns, we propose a generalization of Lemma 2 that would immediately imply Conjecture 1 in its full generality.

Conjecture 2 Let $P$ be a pattern which has a column with a single 1 entry, and let $P^{\prime}$ denote the pattern obtained from $P$ by removing such a column. Then we have

$$
\operatorname{ex}(n, P)=O\left(\operatorname{ex}\left(n, P^{\prime}\right) \log n\right)
$$

Using the fact that every tree has a vertex of degree 1, it would follow from Conjecture 2 that

$$
\mathrm{ex}_{<}(n, H)=O(n \log |V(H)|-3 n)
$$

holds for any ordered tree or forest $H$ whose interval chromatic number is 2 .

In the following two lemmas, we verify Conjecture 2 in some special cases.

Lemma 3 Let $P=\left(p_{i j}\right)$ be a pattern whose $j_{0}$-th column contains a single 1 entry at $p_{i_{0} j_{0}}=1$. Assume further that $p_{i_{0}\left(j_{0}+1\right)}=1$ and that there exists an index $i_{1}$ with $p_{i_{1}\left(j_{0}-1\right)}=p_{i_{1}\left(j_{0}+1\right)}=1$. Let $P^{\prime}$ denote the pattern obtained from $P$ by removing column $j_{0}$. Then we have

$$
\operatorname{ex}(n, P)=O\left(\operatorname{ex}\left(n, P^{\prime}\right) \log n\right)
$$

Proof. Let $A=\left(a_{i j}\right)$ be an $n \times n$ zero-one matrix which avoids $P$ and whose weight is maximum, that is, $w(A)=\operatorname{ex}(n, P)$. For any $i$ and $j$, let $m_{i j}$ stand for the largest $j^{\prime}<j$ with $a_{i j^{\prime}}=1$. In the case when no such $j^{\prime}$ exists, $m_{i j}$ is not defined.

For $0 \leq l \leq\lfloor\log n\rfloor$, define an $n \times n$ zero-one matrix $A_{l}=\left(a_{i j}^{(l)}\right)$, as follows. Set $a_{i j}^{(l)}=1$ if $a_{i j}=1$, $m_{i j}$ is defined, and $j-2^{l+1}<m_{i j} \leq j-2^{l}$. We have that,

$$
\sum_{l=0}^{\lfloor\log n\rfloor} w\left(A_{l}\right) \geq w(A)-n,
$$

as $\sum A_{l}$ contains each 1 entry of $A$ with the exception of the first such entry in each row. Obviously, if $a_{i j}^{(l)}=a_{i j^{\prime}}^{(l)}=1$ and $j<j^{\prime}$, then we have $j+2^{l} \leq j^{\prime}$. Now let $A_{l}^{\prime}$ denote the $n \times n$ zero-one matrix 
obtained by deleting every other 1 entry in every row of $A_{l}$ but keeping $w\left(A_{l}^{\prime}\right) \geq w\left(A_{l}\right) / 2$. Clearly, any two consecutive 1 entries in each row of $A_{l}^{\prime}$ are at least $2^{l+1}$ positions apart.

We claim that, for $0 \leq l \leq\lfloor\log n\rfloor$, the matrix $A_{l}^{\prime}$ avoids $P^{\prime}$. Assume, to the contrary, that $A_{l}^{\prime}$ has a submatrix $B$ which represents $P^{\prime}$. Let column $j_{0}-1$ and column $j_{0}$ of $B$ be columns $j^{\prime}<j^{\prime \prime}$ in $A_{l}^{\prime}$. Let rows $i_{0}$ and $i_{1}$ of $B$ be rows $i^{\prime}$ and $i^{\prime \prime}$ in $A_{l}^{\prime}$. As column $j_{0}$ of $B$ corresponds to column $j_{0}+1$ of $P$, and we have $p_{i_{1}\left(j_{0}-1\right)}=p_{i_{1}\left(j_{0}+1\right)}=p_{i_{0}\left(j_{0}+1\right)}=1$, we obtain that $A_{l}^{\prime}$ has 1 entries in each of the positions $\left(i^{\prime \prime}, j^{\prime}\right),\left(i^{\prime \prime}, j^{\prime \prime}\right)$, and $\left(i^{\prime}, j^{\prime \prime}\right)$. In particular, we have $j^{\prime}+2^{l+1} \leq j^{\prime \prime}$. Now we consider the submatrix $C$ of $A$ consisting of all rows and columns that constitute $B$ and of the additional column $m_{i^{\prime} j^{\prime \prime}}$. As $a_{i^{\prime} j^{\prime \prime}}^{(l)}=1$, the value $m_{i^{\prime} j^{\prime \prime}}$ is well defined and we have $j^{\prime \prime}>m_{i^{\prime} j^{\prime \prime}}>j^{\prime \prime}-2^{l+1} \geq j^{\prime}$. Thus, the new column is column $j_{0}$ of $C$. As $a_{i^{\prime} m_{i^{\prime} j^{\prime \prime}}}=1$, the submatrix $C$ represents $P$, a contradiction.

Now the proof can be completed by simple calculation:

$$
\begin{aligned}
\operatorname{ex}(n, P) & =w(A) \leq n+\sum_{l=0}^{\lfloor\log n\rfloor} w\left(A_{l}\right) \\
& \leq n+2 \sum_{l=0}^{\lfloor\log n\rfloor} w\left(A_{l}^{\prime}\right) \\
& \leq n+2 \sum_{l=0}^{\lfloor\log n\rfloor} \operatorname{ex}\left(n, P^{\prime}\right) \\
& =O\left(\operatorname{ex}\left(n, P^{\prime}\right) \log n\right) .
\end{aligned}
$$

The proof of the following lemma is very similar to that of Lemma 3 and is, therefore, left to the reader.

Lemma 4 Assume that the pattern $P=\left(p_{i j}\right)$ contains two columns $j_{0}$ and $j_{0}+1$, both of which have precisely one 1 entry, at the positions $p_{i_{0} j_{0}}=p_{i_{1}\left(j_{0}+1\right)}=1$. Suppose further that $p_{i_{0}\left(j_{0}-1\right)}=$ $p_{i_{1}\left(j_{0}+2\right)}=1$.

If there exists a row $i_{2}$ with $p_{i_{2}\left(j_{0}-1\right)}=p_{i_{2}\left(j_{0}+2\right)}=1$, then we have

$$
\operatorname{ex}(n, P)=O\left(\operatorname{ex}\left(n, P^{\prime}\right) \log ^{2} n\right),
$$

where $P^{\prime}$ is obtained from $P$ by removing columns $j_{0}$ and $j_{0}+1$.

By multiple application of Theorem 2 and Lemmas 2 and 3, one can easily verify Conjecture 1 for all ordered graphs on at most 6 vertices. For ordered graphs on 7 vertices, we can proceed similarly (also using Lemma 4), except when the adjacency matrix of the forbidden ordered subgraph is equivalent (up to rotation or reflection) to one of the following two patterns:

$$
\left(\begin{array}{llll}
0 & 1 & 0 & 1 \\
1 & 0 & 0 & 1 \\
1 & 0 & 1 & 0
\end{array}\right) \quad\left(\begin{array}{llll}
0 & 1 & 0 & 1 \\
1 & 0 & 1 & 0 \\
1 & 0 & 0 & 1
\end{array}\right)
$$

For these "exceptional" ordered paths of length 6 (a couple of which are depicted in Figure 1), our methods break down. We do not know any upper bound better than $O\left(n^{5 / 3}\right)$, which follows from the fact that the corresponding bipartite graph contains no $K_{3,4}$. 


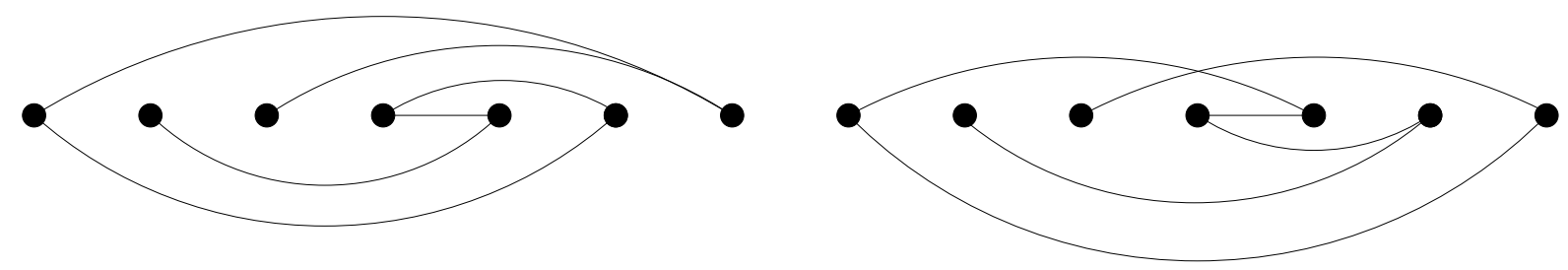

Figure 1.

Two exceptional paths of length 6 .

\section{Cycles}

To formulate our results, we have to consider the following five properties of $n \times N$ zero-one matrices $M$. Properties (a) and (a') correspond to infinite families of forbidden subgraphs, including many ordered cycles. The necessary definitions can be found in the Introduction.

(a) No collection of orthogonal cycles of $M$ is positive.

(a') No orthogonal cycle of $M$ is positive.

(b) $M$ can be obtained from an $n \times N$ real matrix $M^{\prime}=\left(m_{i, j}^{\prime}\right)$ by replacing each 0 entry by 1 and each nonzero entry by 0 . For every $1 \leq i<n$ and $1 \leq j<N$, the matrix $M^{\prime}$ satisfies $d_{i, j}:=m_{i+1, j+1}^{\prime}-m_{i+1, j}^{\prime}-m_{i, j+1}^{\prime}+m_{i, j}^{\prime}>0$.

(b') There is a bivariate twice continuously differentiable real function $f$ satisfying $\frac{d}{d x} \frac{d}{d y} f(x, y)>0$ for all $x$ and $y$ and real values $x_{1}<x_{2}<\ldots<x_{n}, y_{1}<y_{2}<\ldots<y_{N}$ such that $M=\left(m_{i, j}\right)$ is defined by

$$
m_{i, j}= \begin{cases}1 & \text { if } f\left(x_{i}, y_{j}\right)=0 \\ 0 & \text { otherwise }\end{cases}
$$

(c) $M$ can be obtained from a matrix $M^{\prime \prime}$ whose entries are 0,1 , and -1 , by replacing each 0 entry by a 1 and each \pm 1 entry by 0 . Every $2 \times 2$ submatrix $\left(b_{i j}\right)_{i=1,2}^{j=1,2}$ of $M^{\prime \prime}$ satisfies at least one of the following four conditions: $b_{11}=+1, b_{12}=-1, b_{21}=-1$, or $b_{22}=+1$.

We start with the simple connections between the conditions (a) and (a'), and (b) and (b'), respectively.

Lemma 5 For any zero-one matrix $M$, we have

(i) $(a) \Rightarrow\left(a^{\prime}\right)$;

(ii) $(b) \Leftrightarrow\left(b^{\prime}\right)$;

(iii) if $G(M)$ is connected, then $(a) \Leftrightarrow\left(a^{\prime}\right)$.

Proof. We just sketch the simple proofs.

Part (i) is trivial.

For part (ii), (b') $\Rightarrow(\mathrm{b})$ assume $M$ is obtained from the function $f$ as in condition (b'). We define $M^{\prime}=\left(m_{i, j}^{\prime}\right)$ by setting $m_{i, j}^{\prime}=f\left(x_{i}, y_{j}\right)$. Notice that

$$
d_{i, j}=\int_{x_{i}}^{x_{i+1}} \int_{y_{j}}^{y_{j+1}}\left(\frac{d}{d x} \frac{d}{d y} f(x, y)\right) d y d x>0 .
$$


For the reverse implication (b) $\Rightarrow\left(\mathrm{b}^{\prime}\right)$, assume that $M$ can be obtained from the matrix $M^{\prime}=\left(m_{i, j}^{\prime}\right)$ in the way described in condition (b). Set $x_{i}=i$ for $1 \leq i \leq n$ and $y_{j}=j$ for $1 \leq j \leq N$ and define $f_{0}(i, j)=m_{i, j}^{\prime}$ for integers $1 \leq i \leq n$ and $1 \leq j \leq N$. Next we extend $f_{0}$ as a bilinear function to each of the boxes $[i, i+1] \times[j, j+1]$ for integers $1 \leq i<n$ and $1 \leq j<N$, separately. The resulting function $f_{0}$ is continuously defined on $[1, n] \times[1, N]$ and satisfies $\frac{d}{d x} \frac{d}{d y} f(x, y)=d_{i, j}>0$ if $x$ and $y$ are not integers and $i$ and $j$ are their integer parts. However, $f$ is not necessarily differentiable at points with at least one integer coordinate. We define $f$ as a twice continuously differentiable approximation of $f_{0}$ satisfying the condition on the positive mixed derivative everywhere. We can make sure that $f$ agrees with $f_{0}$ on the integer points. Finally, we extend $f$ to the entire real plane keeping the mixed derivative positive everywhere. This function shows that $M$ satisfies $\left(b^{\prime}\right)$.

To establish part (iii) (a') $\Rightarrow(\mathrm{a})$, one has to "combine" the orthogonal cycles of $M$ in a positive collection into one big orthogonal cycle. To combine two orthogonal cycles $C^{\prime}=\left(p_{0}, \ldots, p_{2 k}\right)$ and $C^{\prime \prime}=\left(q_{0}, \ldots, q_{2 l}\right)$ of $M$, consider a sequence of positions $\left(r_{1}, r_{2}, \ldots, r_{2 s}\right)$ that represent a path in $G(M)$ from the vertex corresponding to the row of $p_{0}$ to the vertex corresponding to the row of $q_{0}$. Now $C=\left(p_{0}, p_{1}, \ldots, p_{2 k-1}, r_{1}, r_{2}, \ldots, r_{2 s}, q_{0}, q_{1}, \ldots, q_{2 l-1}, r_{2 s}, r_{2 s-1}, \ldots, r_{1}, p_{0}\right)$ is another orthogonal cycle of $M$ and we have $C(i, j)=C^{\prime}(i, j)+C^{\prime \prime}(i, j)$ for every $i$ and $j$.

The following $6 \times 6$ matrix shows that the $(\mathrm{a}) \Rightarrow\left(\mathrm{a}^{\prime}\right)$ implication cannot be always reversed.

$$
\left(\begin{array}{llllll}
0 & 0 & 1 & 0 & 0 & 1 \\
0 & 0 & 0 & 1 & 1 & 0 \\
1 & 0 & 0 & 0 & 1 & 0 \\
0 & 1 & 0 & 0 & 0 & 1 \\
0 & 1 & 1 & 0 & 0 & 0 \\
1 & 0 & 0 & 1 & 0 & 0
\end{array}\right)
$$

Theorem 4 For any zero-one matrix $M$, conditions (a)-(c) satisfy the following implications: $(a) \Leftrightarrow(b) \Rightarrow(c)$.

Proof. First we show that (b) $\Rightarrow($ a). Assume that an $n \times N$ matrix satisfies condition (b). For any orthogonal cycle $C=\left(p_{0}, \ldots, p_{2 k}\right)$, easy calculation gives

$$
\sum_{i=1}^{n-1} \sum_{j=1}^{N-1} C(i, j) d_{i, j}=\sum_{l=0}^{2 k-1}(-1)^{l} m_{p_{l}}^{\prime},
$$

where $m_{p_{l}}^{\prime}$ represents the entry of the matrix $M^{\prime}$ in position $p_{l}$. If $C$ is an orthogonal cycle of $M$, then the right hand side is clearly 0 . Let $\mathcal{C}$ be a collection of orthogonal cycles of $M$. Summing the above equations we get

$$
\sum_{i=1}^{n-1} \sum_{j=1}^{N-1}\left(\sum_{C \in \mathcal{C}} C(i, j)\right) d_{i, j}=0 .
$$

The linear combination of the positive terms $d_{i, j}$ is zero, therefore one of the coefficients is negative or all are zero. This proves property (a).

(a) $\Rightarrow$ (b) We prove that for an $n \times N$ zero-one matrix $M$ either (b) or the negation of (a) holds. Consider the $n$ by $N$ real matrix $M^{\prime}=\left(m_{i, j}^{\prime}\right)$ that has 0 in place of all 1 entries of $M$ and distinct real variables at all of the remaining positions. Consider the inequalities $d_{i, j}:=m_{i+1, j+1}^{\prime}-m_{i+1, j}^{\prime}-$ $m_{i, j+1}^{\prime}+m_{i, j}^{\prime}>0$ on these variables. The strict linear inequalities determine an open region in the 
variable space, so if this region in nonempty, we can find a solution where no variable is zero. In this case, condition (b) is satisfied.

In the opposite case, when our inequalities do not have a solution, Farkas's lemma states the existence of a positive linear combination of these inequalities yielding $0>0$. Let $k_{i, j} \geq 0$ be the coefficient of $d_{i, j}>0$ in such a linear combination. We can assume that these coefficients are integers, so that $k_{i, j}$ is a nonnegative integer for all $i$ and $j$, and not all of them are zero. We define $k_{i, j}=0$ if $i=0$ or $n$, or if $j=0$ or $N$.

We build an oriented multigraph $G^{*}$ on the vertex set $\left\{v_{i, j}: 1 \leq i \leq n, 1 \leq j \leq N\right\}$ as follows. There are two types of edges in $G^{*}$ : For any $1 \leq i \leq n, 1 \leq j<N$, we connect $v_{i, j}$ and $v_{i, j+1}$ by $\left|k_{i-1, j}-k_{i, j}\right|$ horizontal edges. If $k_{i-1, j}>k_{i, j}$, these edges are directed toward $v_{i, j}$, otherwise they are directed toward $v_{i, j+1}$. Similarly, for any $1 \leq i<n, 1 \leq j \leq N, v_{i, j}$ and $v_{i+1, j}$ are connected by $\left|k_{i, j-1}-k_{i, j}\right|$ vertical edges directed toward $v_{i+1, j}$ or $v_{i, j}$, depending on whether $k_{i, j-1}>k_{i, j}$ or the other way around. It is easy to verify that in this graph every vertex has the same indegree and outdegree. Therefore, the edge set of $G^{*}$ can be partitioned into directed cycles. Whenever $M$ has a zero at a position $(i, j)$, we know that the variable $m_{i, j}^{\prime}$ will cancel at the combination $\sum k_{i, j} d_{i, j}$. This implies that the numbers of incoming and outgoing horizontal edges incident to any such vertex $v_{i, j}$ must coincide. Therefore, the cycles of the edge partition can be chosen so that none of them "bends" at such vertices, i.e., all of their bends occur at positions where $M$ has an entry 1. We cannot exclude self-crossing cycles that pass through the same vertex more than once. The orthogonal cycles corresponding to edge partitions with the above property are orthogonal cycles of $M$. Moreover, it is easy to argue that they form a collection $\mathcal{C}$ that satisfies $\sum_{C \in \mathcal{C}} C(i, j)=k_{i, j}$ for all $i$ and $j$. This shows that condition (a) does not hold for $M$.

(b) $\Rightarrow$ (c) Suppose that $M$ can be obtained from $M^{\prime}=\left(m_{i, j}^{\prime}\right)$ in the way described in (b), and define a matrix $M^{\prime \prime}=\left(m_{i, j}^{\prime \prime}\right)$ by setting $m_{i, j}^{\prime \prime}=\operatorname{sign}\left(m_{i, j}^{\prime}\right)$. Consider the submatrix of $M^{\prime \prime}$ defined by the rows $i_{1}<i_{2}$ and columns $j_{1}<j_{2}$. We have $m_{i_{2}, j_{2}}^{\prime}-m_{i_{1}, j_{2}}^{\prime}-m_{i_{2}, j_{1}}^{\prime}+m_{i_{1}, j_{1}}=\sum_{i=j_{1}}^{j_{2}-1} \sum_{j=j_{1}}^{j_{2}-1} d_{i, j}>0$. This implies that at least one of the following conditions must be satisfied: $m_{i_{1}, j_{1}}^{\prime}$ or $m_{i_{2}, j_{2}}^{\prime}$ is positive, or $m_{i_{1}, j_{2}}^{\prime}$ or $m_{i_{2}, j_{1}}^{\prime}$ is negative.

We remark that condition (a'), which is somewhat weaker than (a), also implies (c) as can be shown by constructing the corresponding matrix $M^{\prime \prime}$ entry by entry. The following $6 \times 6$ matrix satisfies condition (c) for $M^{\prime \prime}$, but the corresponding matrix $M$ does not have property (a). Thus, the implication $(\mathrm{b}) \Rightarrow(\mathrm{c})$ cannot be always reversed.

$$
\left(\begin{array}{cccccc}
0 & + & + & 0 & + & + \\
- & 0 & 0 & - & + & + \\
- & 0 & - & - & - & 0 \\
- & + & 0 & - & 0 & + \\
+ & + & + & 0 & 0 & + \\
0 & + & - & - & - & 0
\end{array}\right)
$$

Theorem 5 (i) The maximum weight of an $n \times n$ zero-one matrix with property $(c)$ is $O\left(n^{4 / 3}\right)$.

(ii) For arbitrarily large values of $n$, there exist $n \times n$ zero-one matrices of weight $\Omega\left(n^{4 / 3}\right)$ that satisfy condition ( $\left.b^{\prime}\right)$ (and thus conditions ( $\left.a\right),\left(a^{\prime}\right),(b)$, and (c) are also satisfied).

Proof. (i) Let $M$ be an $n \times n$ zero-one matrix satisfying condition $(c)$, and let $M^{\prime \prime}=\left(m_{i j}^{\prime \prime}\right)$ be the corresponding matrix with $-1,0$, and 1 entries. 
For a fixed $0 \leq i \leq n$, we define a linear ordering on the symbols $p_{i j}$, where $1 \leq j \leq n$. For $1 \leq j<j^{\prime} \leq n$, set $p_{i j^{\prime}}<p_{i j}$ if there exists a row $1 \leq i^{\prime} \leq i$ with $m_{i^{\prime} j}^{\prime \prime} \geq 0$ and $m_{i^{\prime} j^{\prime}}^{\prime \prime} \leq 0$. Otherwise, set $p_{i j}<p_{i j^{\prime}}$.

To see that this definition indeed gives rise to a linear order, we have to check that for $1 \leq j<$ $j^{\prime}<j^{\prime \prime} \leq n$ we cannot have $p_{i j}<p_{i j^{\prime}}<p_{i j^{\prime \prime}}<p_{i j}$, nor can it occur that $p_{i j}<p_{i j^{\prime \prime}}<p_{i j^{\prime}}<p_{i j}$. To exclude the first possibility, assume $p_{i j^{\prime \prime}}<p_{i j}$. Then there exists $1 \leq i^{\prime} \leq i$ such that $m_{i^{\prime} j}^{\prime \prime} \geq 0$ and $m_{i^{\prime} j^{\prime \prime}}^{\prime \prime} \leq 0$. If $m_{i^{\prime} j^{\prime}}^{\prime \prime} \geq 0$, then we have $p_{i j^{\prime \prime}}<p_{i j^{\prime}}$, while if $m_{i^{\prime} j^{\prime}}^{\prime \prime} \leq 0$, it follows that $p_{i j^{\prime}}<p_{i j}$. In either case, we obtain a contradiction.

To exclude the second possibility assume that $p_{i j^{\prime \prime}}<p_{i j^{\prime}}<p_{i j}$. Then there exist suitable indices $1 \leq i^{\prime} \leq i$ and $1 \leq i^{\prime \prime} \leq i$ such that $m_{i^{\prime} j}^{\prime \prime} \geq 0, m_{i^{\prime} j^{\prime}}^{\prime \prime} \leq 0, m_{i^{\prime \prime} j^{\prime}}^{\prime \prime} \geq 0$, and $m_{i^{\prime \prime} j^{\prime \prime}}^{\prime \prime} \leq 0$. We claim that for $i^{*}=\max \left(i^{\prime}, i^{\prime \prime}\right)$ we have $m_{i^{*} j}^{\prime \prime} \geq 0$ and $m_{i^{*} j^{\prime \prime}}^{\prime \prime} \leq 0$, and hence $p_{i j^{\prime \prime}}<p_{i j}$, which is a contradiction. This claim is trivial for $i^{\prime}=i^{\prime \prime}$. If $i^{\prime}<i^{\prime \prime}$, the claim follows from condition (c) applied to the submatrix determined by rows $i^{\prime}$ and $i^{\prime \prime}$ and columns $j$ and $j^{\prime}$. If $i^{\prime}>i^{\prime \prime}$, it follows from condition (c) applied to the submatrix determined by rows $i^{\prime}$ and $i^{\prime \prime}$ and columns $j^{\prime}$ and $j^{\prime \prime}$.

Let us represent $p_{i j}(0 \leq i \leq n, 1 \leq j \leq n)$ by points in the plane, denoted by the same symbols. For a fixed $i$, we choose the points $p_{i j}$ on the line $y=i$, ordered from left to right according to the linear order defined above. For $1 \leq j \leq n$, we draw a $y$-monotone curve $l_{j}$ connecting the points $\left\{p_{i j}\right\}_{i=0}^{n}$. This can be done in a such a way that $l_{j}$ and $l_{j^{\prime}}$ cross at most once between the horizontal lines $y=i-1$ and $y=i$. Moreover, such a crossing occurs if and only if the order of $p_{(i-1) j}$ and $p_{(i-1) j^{\prime}}$ is different from that of $p_{i j}$ and $p_{i j^{\prime}}$.

It is clear from the definition that if $p_{i j^{\prime}}<p_{i j}$ for some $0 \leq i \leq n$ and $1 \leq j<j^{\prime} \leq n$, then we also have $p_{i^{\prime} j^{\prime}}<p_{i^{\prime} j}$ for all $i<i^{\prime} \leq n$. Thus, the total number of intersections between the curves $l_{j}$ and $l_{j^{\prime}}$ is at most one. In other words, these curves form a collection of pseudolines.

For any $1 \leq i \leq n$, consider the set of indices $J_{i}=\left\{j \mid m_{i j}=0\right\}$. Let $j, j^{\prime} \in J_{i}, j<j^{\prime}$. By the definition of the ordering, it is clear that $p_{i j^{\prime}}<p_{i j}$. On the other hand, it follows from condition (c) that $p_{(i-1) j}<p_{(i-1) j^{\prime}}$. Thus, the pseudolines $l_{j}, j \in J_{i}$ must pairwise cross each other between the horizontal lines $y=i-1$ and $y=i$. Modifying these pseudolines within the horizontal strip $i-1<y<i$, we can make sure that all of them pass through the same point $P_{i}$. Thus, we obtain a collection of $n$ pseudolines $l_{j}$ and a set of $n$ points $P_{i}$ in the plane. The number of point-pseudoline incidences between them is exactly the same as the number of 1 entries in the matrix $M$. According to the generalization of the Szemerédi-Trotter theorem [14] by Clarkson et al. and Székely [6, 13], the number of incidences between $n$ points and $n$ pseudolines is $O\left(n^{4 / 3}\right)$, which proves part (i).

(ii) Consider a collection of $n$ straight lines and $n$ points in the plane with $\Omega\left(n^{4 / 3}\right)$ incidences between them. Assume that all points have distinct $x$ coordinates, all lines have distinct slopes, and none of them is vertical. The standard example of a point set and a line set with many incidences is an $\sqrt{n} \times \sqrt{n}$ integer grid with the $n$ lines containing the highest number of points. There are many parallel lines in this example, but we can get rid of them (along with the vertical lines and the points with identical $x$ coordinates) using a generic projective linear transformation that keeps the number of incidences unchanged. Denote the points by $P_{i}=\left(x_{i}, v_{i}\right)$ with $x_{1}<x_{2}<\ldots<x_{n}$, and the lines by $l_{i}: y=y_{i} x+w_{i}$ with $y_{1}<y_{2}<\ldots<y_{n}$.

Let

$$
f(x, y)=x y-f_{1}(x)+f_{2}(y),
$$

where $f_{1}$ and $f_{2}$ are twice continuously differentiable functions such that $f_{1}\left(x_{i}\right)=v_{i}$ and $f_{2}\left(y_{i}\right)=w_{i}$. Clearly, we have $\frac{d}{d x} \frac{d}{d y} f(x, y)=1>0$. Furthermore, defining the matrix $M=\left(m_{i j}\right)$ as in condition (b'), we have $m_{i j}=1$ if and only if $f\left(x_{i}, y_{j}\right)=0$, which happens if and only if $P_{i}$ is incident to $l_{j}$. 
Thus, the weight of $M$ satisfies $w(M)=\Omega\left(n^{4 / 3}\right)$, as required.

\section{Geometric consequences and concluding remarks}

A. First we deduce Corollary 1, the best known bound on the number of unit distances determined by $n$ points in the plane, from Theorem 5 .

Proof of Corollary 1. Let $P$ be a set of $n$ points in the plane. Let $l$ be a line in general position; i.e., assume that $l$ does not pass through any point in $P$ and that the orthogonal projections of the elements of $P$ onto $l$ are all distinct. Let $l$ partition the point set $P$ into two subsets, $P_{1}$ and $P_{2}$, containing $n_{1}$ and $n_{2}$ elements, respectively, where $n_{1}+n_{2}=n$. Construct an $n_{1} \times n_{2}$ matrix $A=\left(a_{p q}\right)$, as follows. Let the rows (and columns) of $A$ correspond to the points of $P_{1}$ (and $P_{2}$, respectively), in the order of their projections to $l$. Let the entry $a_{p q}$ in the row of $A$ corresponding to $p \in P_{1}$ and in the column corresponding to $q \in P_{2}$ depend on the Euclidean distance $d(p, q)$ between $p$ and $q$ :

$$
a_{p q}=\left\{\begin{array}{rll}
-1 & \text { if } & d(p, q)<1 \\
0 & \text { if } & d(p, q)=1 \\
1 & \text { if } & d(p, q)>1
\end{array}\right.
$$

We claim that $M^{\prime \prime}:=A$ satisfies the requirement in condition (c) formulated at the beginning of Section 4. To see this, assume without loss of generality that $l$ is horizontal and let $p, q, r$, and $s$ be points in $P$ with $p$ and $q$ above $l$, and $r$ and $s$ below $l$. Furthermore, let $q$ be to the right of $p$, and let $s$ be to the right of $r$. We need to show that at least one of the following four inequalities are valid: $d(p, r)<1, d(p, s)>1, d(q, r)>1, d(q, s)<1$. (See Figure 2.) Indeed, if the four points form a convex quadrilateral in the order $p q s r$, then this follows from the fact, that the sum of the lengths of its two diagonals is larger than the total lengths of two opposite edges: $d(p, s)+d(q, r)>d(p, r)+d(q, s)$. If this is not the case, then $p$ or $q$ is not above the line $r s$, or $r$ or $s$ is not below the line $p q$. Let us assume that $p$ or $q$ is not above the line $r s$. Then $r s$ must intersect $l$ at a point $x$. Point $x$ is either to the left of $r$ or to the right of $s$. Assume without loss of generality that the first possibility holds. Then $p$ or $q$ is to the left of $x$, so $p$ (which is to the left of $q$ ) must be in the quadrant to the left of $x$ and above $l$. This implies $d(p, r)<d(p, s)$, so we have $d(p, r)<1$ or $d(p, s)>1$.
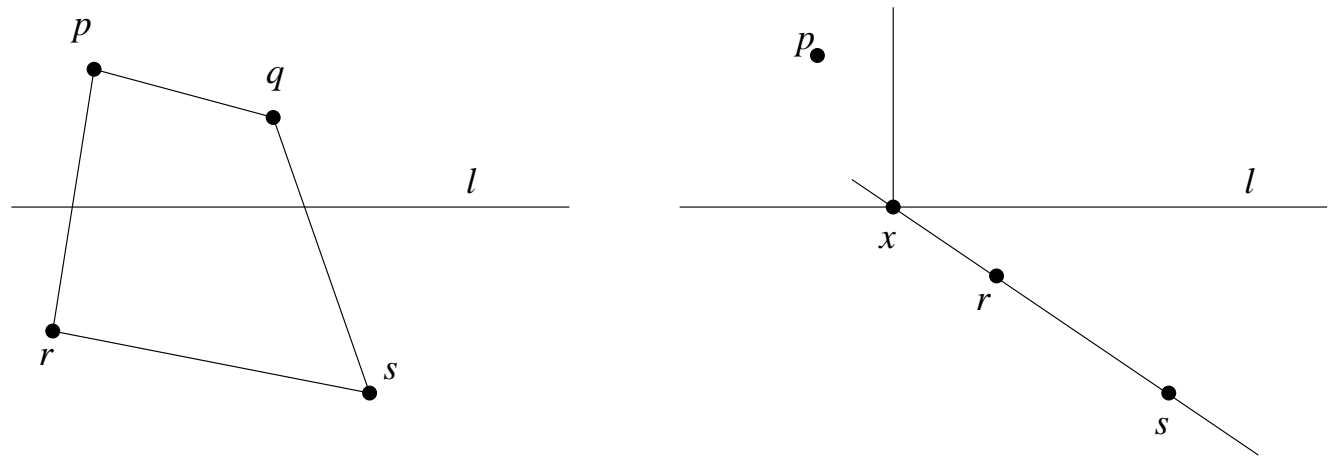

Figure 2.

We can apply Theorem 5 (i) to conclude that $A$ has $O\left(n^{4 / 3}\right)$ zero entries. In other words, the number of pairs of points that determine distance one and are separated by the line $l$ is at most 
$O\left(n^{4 / 3}\right)$.

We finish the proof by choosing a random direction and, again randomly, placing infinitely many parallel lines in the chosen direction so that the distance between any two consecutive lines is 2 . Let $\mathcal{L}$ denote the family of selected lines. For any $l \in \mathcal{L}$, let $P_{l}$ denote the set of points $p \in P$ within distance 1 of $l$. All the unit distance pairs of point in $P$ that $l$ separates are in $P_{l}$, so $l$ separates $O\left(\left|P_{l}\right|^{4 / 3}\right)$ pairs.

As the sets $P_{l}$ are disjoint we have $\sum_{l \in \mathcal{L}}\left|P_{l}\right| \leq n$. The total number of unit distance pairs separated by a member of $\mathcal{L}$ is $\sum_{l \in \mathcal{L}} O\left(\left|P_{l}\right|^{4 / 3}\right)=O\left(n^{4 / 3}\right)$. Since each unit distance pair of points has a positive constant chance of being separated by a member of $\mathcal{L}$, the result follows.

Note that the above argument does not use any specific property of the Euclidean norm. For any strictly convex norm $N$, one can slightly modify the proof to show that a set $P$ of $n$ points in the plane has $O\left(n^{4 / 3}\right)$ pairs at $N$-distance 1 . Let $l$ be a line in general position that splits $P$ into two parts $P_{1}$ and $P_{2}$. Consider a unit circle with respect to the $N$-norm centered at a point of $l$, and take a tangent $t$ to this circle at one of its intersection points with $l$. Order the elements of $P_{1}$ and $P_{2}$ according to their projections onto $l$, parallel to $t$. Define the matrix $A=\left(a_{p q}\right)$ for $p \in P_{1}, q \in P_{2}$ by letting $a_{p q}=\operatorname{sign}\left(d_{N}(p, q)-1\right)$. As in the Euclidean case, one can show that $A$ meets the requirements on $M^{\prime \prime}$ in condition (c). Thus, Theorem 5 implies that the number of unit- $N$-distance pairs in $P$ separated by $l$ is $O\left(n^{4 / 3}\right)$. We proceed by randomly choosing a direction and, again randomly, placing infinitely many lines in this direction such that the $N$-distance between any pair of consecutive lines is two. The number of unit- $N$-distance point pairs in $P$ separated by at least one of these lines is still $O\left(n^{4 / 3}\right)$. The probability that a segment of $N$-distance one is cut by a line belonging to the family is bounded from below by a positive constant depending on $N$. Hence, the number of unit- $N$-distance pairs is $O\left(n^{4 / 3}\right.$, where the constant of proportionality depends on $N$. We can get rid of the dependence on $N$ by first applying an affine transformation that brings the norm $N$ close to the Euclidean norm. In other words, we can assume without loss of generality that $1 \leq d_{N}(x, y) / d(x, y) \leq 2$ for all points $x \neq y$. Now the probability that a unit- $N$-distance pair is separated by one of the lines in our random collection is bounded from below by a positive absolute constant. Thus, with respect to any strictly convex norm, the number of unit distance pairs determined by a set of $n$ points in the plane is $O\left(n^{4 / 3}\right)$, where the constant of proportionality does not depend on $N$.

According to Brass [4] and Valtr [16], there exist strictly convex norms with respect to which the maximal number of unit distances among $n$ points in the plane is $\Theta\left(n^{4 / 3}\right)$. One can only hope to make further progress in bounding the number of unit distance pairs by finding forbidden patterns characteristic of the Euclidean norm.

This is the first proof of this result, that does not use any combinatorial tool other than a "forbidden pattern" argument. Our proof cannot be considered entirely independent, because the proof of Theorem 5 was based on Székely's $O\left(n^{4 / 3}\right)$ upper bound on the number of incidences between $n$ points and $n$ pseudolines in the plane, from where one can directly deduce Corollary 1.

Note that Theorem 5 involves an infinite class of forbidden patterns. It would be interesting to come up with an alternative argument using only a finite number of forbidden configurations, perhaps only 4-cycles and noncrossing hexagons.

B. For any unordered graph $H_{0}$, let $\operatorname{ex}_{0}\left(n, H_{0}\right)$ stand for the maximal number of edges that a simple unordered graph with $n$ vertices can have if it does not contain $H_{0}$ as a (not necessarily induced) subgraph. 
Füredi and Hajnal [9] conjectured that for every ordered graph $H$ with interval chromatic number 2 , the extremal function $\operatorname{ex}(n, A(H))$ is close to $\operatorname{ex}_{0}\left(n, H_{0}\right)$, where $H_{0}$ denotes the unordered graph obtained from $H$ by disregarding the ordering of the vertices. More precisely, they asked whether

$$
\operatorname{ex}(n, A(H))=O\left(\operatorname{ex}_{0}\left(n, H_{0}\right) \log n\right)
$$

holds for all ordered graphs $H$ with $\chi_{<}(H)=2$.

We answer this question in the negative. Let $H$ be any even cycle of length $k \geq 8$, whose vertices are ordered in such a way that $\chi_{<}(H)=2$ and the 1 entries of $A(H)$ form a positive orthogonal cycle. (It is easy to see that such an ordering exists.) Obviously, no matrix satisfying condition (a) or (a') can contain $A(H)$. By Theorem 5 (ii), there exist $n \times n$ zero-one matrices of weight $\Omega\left(n^{4 / 3}\right)$ which satisfy condition (a). Thus, we have $\operatorname{ex}(n, A(H))=\Omega\left(n^{4 / 3}\right)$. On the other hand, $H_{0}=C_{k}$ and, by the Bondy-Simonovits theorem (see [3]), we have $\operatorname{ex}_{0}\left(n, C_{k}\right)=O\left(n^{1+\frac{2}{k}}\right)$. For $k \geq 8$, these two bounds are far apart.

Let us remark that Füredi and Hajnal, perhaps having doubts about their conjecture, also asked if their statement holds at least for trees. This problem is still open and it can be regarded as a strong version of our Conjecture 1.

C. Braß, Károlyi, and Valtr [5] studied cyclically ordered graphs and asked whether the vertices of every graph $H_{0}$ can be cyclically ordered so that the extremal functions of the unordered and ordered graphs differ by at most a constant factor. Without precisely defining cyclically ordered (in their terminology, "convex geometric") graphs, we note that their conjecture would immediately imply that the vertices of any connected bipartite graph $H_{0}$ can be ordered in such a way that the resulting ordered graph $H$ has interval chromatic number 2 and satisfies

$$
\operatorname{ex}\left(n,\left\{A(H),(A(H))^{T}\right\}\right)=O\left(\operatorname{ex}_{0}\left(n, H_{0}\right)\right) .
$$

Our counterexample to this conjecture is a tree of seven vertices: let $H_{0}$ consist of three paths of length 2, joined at a common endpoint. Since $H_{0}$ is a tree, we have $\operatorname{ex}_{0}\left(n, H_{0}\right)=O(n)$. It is easy to see that for any ordering $H$ of $H_{0}$ of interval chromatic number 2 the matrix $A(H)$ contains the pattern $F=\left(\begin{array}{lll}1 & 1 & 0 \\ 1 & 0 & 1\end{array}\right)$ or one of the seven other patterns obtainable from $F$ by rotation or reflection. If $A(H)$ contains $F$, then $\operatorname{ex}\left(n,\left\{A(H),(A(H))^{T}\right\}\right) \geq \operatorname{ex}\left(n,\left\{F, F^{T}\right\}\right)$, where the latter extremal function is $\Theta(n \log n)$ as proved in both of the papers [2,8]. By symmetry we have to consider only one more pattern: let us assume $A(H)$ contains $F_{1}=\left(\begin{array}{lll}1 & 0 & 1 \\ 1 & 1 & 0\end{array}\right)$. In this case we have $\operatorname{ex}\left(n,\left\{A(H),(A(H))^{T}\right\}\right) \geq \operatorname{ex}\left(n,\left\{F_{1}, F_{1}^{T}\right\}\right)$. Here we also have $\operatorname{ex}\left(n,\left\{F_{1}, F_{1}^{T}\right\}\right)=\Theta(n \log n)$ as proved in [15]. (An earlier lower bound of $\Omega(n \log n / \log \log n$ ) is proved in [2].) In neither case does the required inequality hold: the left-hand side is larger than the right-hand side by a factor of $\log n$.

D. It is tempting to make the following "optimistic" conjecture that can be regarded as the "least common denominator" of the two conjectures disproved above.

Conjecture 3 The vertices of any unordered graph $H_{0}$ can be ordered in such a way that for the resulting ordered graph $H$ we have $\operatorname{ex}_{<}(n, H)=O\left(\operatorname{ex}_{0}\left(n, H_{0}\right) \log n\right)$.

Acknowledgement. We are very grateful to our friend, Rom Pinchasi, for many important observations and valuable insights that led to the birth of this paper. 


\section{References}

[1] N. Alon and E. Friedgut: On the number of permutations avoiding a given pattern, J. Combin. Theory Ser. A 89 (2000), 133-140.

[2] D. Bienstock and E. Győri: An extremal problem on sparse 0-1 matrices, SIAM Journal on Discrete Mathematics 4 (1991), 17-27.

[3] B. Bollobás: Extremal Graph Theory, Academic Press, London, 1978.

[4] P. Brass: On lattice polyhedra and pseudocircle arrangements, in Charlemagne and his Heritage-1200 Years of Civilization and Science in Europe, Vol. 2: Mathematical Arts, P.L. Butzer et al., eds., Brepols Verlag 1988, 297-302.

[5] P. Braß, Gy. Károlyi, and P. Valtr: A Turán-type extremal theory of convex geometric graphs, Discrete and Computational Geometry-The Goodman-Pollack Festschrift (B. Aronov et al., eds.), Springer-Verlag, Berlin, 2003, 277-302.

[6] K. Clarkson, H. Edelsbrunner, L. Guibas, M. Sharir, and E. Welzl: Combinatorial complexity bounds for arrangements of curves and spheres, Discrete Comput. Geom. 5 (1990), 99-160.

[7] P. Erdős and A.H. Stone: On the structure of linear graphs, Bull. Amer. Math. Soc. 52 (1946), 1087-1091.

[8] Z. Füredi: The maximum number of unit distances in a convex n-gon, Journal of Combinatorial Theory Series A 55 (1990), 316-320.

[9] Z. Füredi and P. Hajnal: Davenport-Schinzel theory of matrices, Discrete Mathematics 103 (1992), 233-251.

[10] N.H. Katz: On the self crossing six sided figure problem, New York J. Math. 5 (1999), 121-130.

[11] A. Marcus and G. Tardos: Excluded permutation matrices and the Stanley-Wilf conjecture, Journal of Combinatorial Theory, Series A 107 (2004), 153-160.

[12] J. Spencer, E. Szemerédi, and W.T. Trotter: Unit distances in the Euclidean plane, in: Graph Theory and Combinatorics (B. Bollobás, ed.), Academic Press, New York, 1984, 293-303.

[13] L.A. Székely: Crossing numbers and hard Erdős problems in discrete geometry, Combinatorics, Probability, and Computing 6 (1997), 353-358.

[14] E. Szemerédi and W.T. Trotter, Jr.: Extremal problems in discrete geometry, Combinatorica 3 (1983), 381-392.

[15] G. Tardos: On 0-1 matrices and small excluded submatrices, Journal of Combinatorial Theory, Series A, to appear.

[16] P. Valtr: Strictly convex norms allowing many unit distances, manuscript. 